\title{
Correlation of Choroidal Thickness with Age in Healthy Subjects - Automatic Detection and Segmentation using A Deep Learning Model
}

Chen Yu Lin ( $\nabla$ cylinscholar@gmail.com )

Taichung Veterans General Hospital

\section{Yu Len Huang}

Tunghai University

Wei Ping Hsia

Taichung Veterans General Hospital

\section{Yang Wang}

Tunghai University

\section{Chia-Jen Chang}

Taichung Veterans General Hospital

\section{Research Article}

Keywords: Deep learning, Mask region-based convolutional neural network, Optical coherence tomography, Choroidal thickness, Automatic segmentation

Posted Date: November 29th, 2021

DOI: https://doi.org/10.21203/rs.3.rs-1071924/v1

License: (c) (1) This work is licensed under a Creative Commons Attribution 4.0 International License.

Read Full License

Version of Record: A version of this preprint was published at International Ophthalmology on April 5th, 2022. See the published version at https://doi.org/10.1007/s10792-022-02292-8. 


\section{Abstract}

Propose: The proposed deep learning model with a mask region-based convolutional neural network (Mask R-CNN) can predict choroidal thickness automatically. Changes in choroidal thickness with age can be detected with manual measurements. In this study, we aimed to investigate choroidal thickness in healthy eyes by utilizing the Mask R-CNN model.

Methods: A total of 68 eyes from 57 participants without significant ocular disease were recruited. The participants were allocated to one of three groups according to their age and underwent spectral domain optical coherence tomography (SD-OCT) centered on the fovea. Physicians labelled the choroidal contours in all the OCT sequences. We applied the Mask R-CNN model for automatic segmentation. Comparisons of choroidal thicknesses were conducted according to age and prediction accuracy.

Results: Older age groups had thinner choroids, according to the automatic segmentation results; the mean choroidal thickness was $253.7 \pm 41.9 \mu \mathrm{m}$ in the youngest group, $206.8 \pm 35.4 \mu \mathrm{m}$ in the middleaged group, and $152.5 \pm 45.7 \mu \mathrm{m}$ in the oldest group $(p<0.01)$. Measurements obtained using physician sketches demonstrated similar trends. We observed a significant negative correlation between choroidal thickness and age $(p<0.01)$. The prediction error was lower and less variable in choroids that were thinner than the cutoff point of $280 \mu \mathrm{m}$.

Conclusion: The mean choroidal thickness decreased with age in healthy subjects. The Mask R-CNN model can accurately predict choroidal thickness, especially choroids thinner than $280 \mu \mathrm{m}$. This model can enable exploring larger and more varied choroid datasets comprehensively, automatically, and conveniently.

\section{Introduction}

The choroid, located underneath the retina and above the sclera, is the posterior portion of the uveal tract. It comprises numerous blood vessels and pigmented stroma. The choroid is vital for visual physiology. For example, it supplies nutrients and oxygen to the outer retina, including the retinal pigment epithelium (RPE) and photoreceptors; moreover, choroidal melanocytes absorb excess light [1, 2].

Pathological changes in the choroid are related to many vision-threatening diseases, such as age-related macular degeneration (AMD), polypoidal choroidal vasculopathy (PCV), central serous chorioretinopathy (CSCR), pathologic myopia, and autoimmune disease; choroidal thickness is also associated with these diseases [3-6]. Detecting the choroid and estimating its thickness can facilitate clinical diagnosis and disease progression monitoring tasks [7]. Previous studies have reported that choroidal thickness is negatively correlated with age in healthy eyes, not only in terms of the subfoveal choroidal thickness (SFCT), but also in relation to the thickness over the nasal site and temporal site [8-10].

Optical coherence tomography (OCT) is a convenient approach to identifying and measuring the structure of the posterior visual segment, which can provide details of the retinal and choroidal layers. Owing to 
technological advancements and population aging, OCT is becoming increasingly essential for diagnosing many ocular diseases. Accordingly, an appropriate model for automatic recognition of features in medical images is crucial. For instance, a choroid segmentation model can aid ophthalmologists in accurate clinical diagnoses and effective management as well as in the development of a database or comparison site for different groups. In addition, manually outlining the boundary of the choroid is a time-consuming task, and it can be challenging when the borders are ambiguous. Therefore, developing an automatic OCT image analysis and segmentation model is crucial.

Depicting the choroid border is complex, especially at the choroidal-scleral interface (CSI), because of the inconsistent and inhomogeneous tissue texture. In the past decade, scholars have proposed several automatic models for segmenting the choroid from OCT images. For example, Tian et al. presented a mathematical algorithm for detecting Bruch's membrane by searching for the pixel with the largest gradient value near the RPE and delineating the CSI using the Dijkstra algorithm [11]. This algorithm is implemented semiautomatically, requiring time-consuming manual sketching tasks. Deep learning models are gradually being utilized in automatic feature recognition in choroidal images. Masood et al. and $\mathrm{He}$ et al. have proposed approaches based on a combination of morphological and deep learning methodologies involving convolutional neural network (CNN) $[12,13]$. They developed CNN classifiers and a I2-Iq $(0<\mathrm{q}<1)$ fitter model based on the LeNet-5 framework; their classifiers can delineate the outer boundary of the choroid, but the convolutional layers can extract only shallow features. Mask regionbased CNN (Mask R-CNN), a classic neural network algorithm for instance segmentation, constitutes a powerful model that can complete object detection, classification, and instance segmentation simultaneously [14]. Hsia et al. proposed a Mask R-CNN model for the automatic segmentation of the choroid. They observed that their model could preserve crucial information on location, including shallow and deep layer features, and that it could achieve an accurate prediction rate and faster choroidal boundary segmentation [15]. Overall, Mask R-CNN models are accurate and effective in the automatic segmentation of choroid images.

Previous studies evaluating choroidal thickness in different age groups have mainly relied on discontinuous measurements at different sites of the choroid $[9,10,16,17]$. An automatic segmentation model might provide continuous measurements and more comprehensive quantifications of choroidal thickness.

Accordingly, we investigated the choroidal thickness in healthy subjects from different age groups in Taiwan by using the deep learning method with the Mask R-CNN model.

\section{Materials And Methods}

\subsection{Participants and clinical evaluation}

We recruited participants of different ages from Taichung Veterans General Hospital, Taiwan. The inclusion criterion was having a refractive error, measured as the spherical equivalent (SE), of between 
-6.0 and +6.0 D; we used this criterion to reduce the possible influence of high myopia, high hyperopia, and axial length. We excluded individuals with ocular diseases that would potentially influence the appearance and shape of the retina and choroid, such as ocular tumors, autoimmune diseases, uveitis, subretinal fluid, or large drusens. We also excluded individuals who had previously received intraocular interventions, such as vitrectomy or intravitreal injection. Accordingly, this study included 57 healthy subjects aged from 21 to 79 years (total eyes: 68).

\subsection{Data compilation}

We applied an OCT image dataset composed of 68 eyes from 57 participants. All the participants underwent a spectral domain OCT (SD-OCT) scan (Heidelberg Engineering, Heidelberg, Germany). The quality and depth of these images were confirmed by physicians and were considered adequate for the visualization and analysis of the choroid. Each OCT scan sequence comprised 25 two-dimensional slices covering a $6 \times 6 \mathrm{~mm}$ area surrounding the center of the fovea. The interval between consecutive slices was $24 \mu \mathrm{m}$. The spatial resolution of each image was 1,008 $\times 596$ pixels, with 1 pixel corresponding to 4 $\mu \mathrm{m}$. Each OCT image was monochrome with 256 gray levels. In addition, we removed any dispensable parts of the image by clipping every slice into a $480 \times 480$ pixel format. Physicians outlined the contours of the choroids in all OCT scan sequences by using LabelMe [18]. We judged the CSI to be the outer border of the choroid, and we considered Bruch's membrane to represent the inner border.

\subsection{Segmentation using Mask R-CNN model}

We used Mask R-CNN model for choroid detection. The model involves a two-stage framework. In the first stage, an image is convolved to generate distinguishable feature maps and recommendations through a regional proposal network (RPN). In the second stage, the proposals are classified and bounding boxes and masks are generated. The proposed model is based on a deep residual network (ResNet) and a feature pyramid network (FPN). The Mask R-CNN model has standard convolutional and fully connected layers, which are used for mask generation and object detection.

The proposed model receives an OCT image and outputs a mask of the choroid. The Mask R-CNN model involves three steps. First, a $480 \times 480$ pixel region-of-interest image is extracted and then used in the training network to extract features and generate a feature map. Second, the generated feature map is sent to the RPN to locate proposals related to the choroid. Third, the proposals are subjected to classification and regression processes, after which the fully connected network (FCN) is implemented to generate a mask for the choroid. Figure 1 illustrates the framework of the proposed Mask R-CNN model.

\subsection{Statistical analysis}

We used SPSS (version 20) for our statistical analyses. We applied an analysis of variance (ANOVA) to compare variables between the different age groups. Spearman's correlation and linear regression were applied to examine the relationship between age, SE, and choroidal thickness. Data are presented as mean \pm standard deviation. Statistical significance was defined as a two-tailed $p$ value of less than 0.05 for all statistical analyses. 


\section{Results}

We included a total of 68 eyes from 28 men and 29 women in this study. We divided the participants into 20-39-, 40-59-, and 60-79-year age groups, comprising 19, 22, and 27 eyes, respectively. Table 1a presents the demographic and clinical characteristics of the different age groups.

Table 1

a. Demographic and clinical characteristics of participants

\begin{tabular}{|lllll|}
\hline $\begin{array}{l}\text { Age group } \\
\text { (years) }\end{array}$ & $\begin{array}{l}\text { Mean age } \\
\text { (years) }\end{array}$ & $\begin{array}{l}\text { Mean SE } \\
\text { (diopters) }\end{array}$ & $\begin{array}{l}\text { Number of eyes } \\
\text { (subjects) }\end{array}$ & $\begin{array}{l}\text { number of } \\
\text { OD/OS }\end{array}$ \\
\hline $20-39$ & $29.7 \pm 5.9$ & $-1.2 \pm 0.7$ & $19(16)$ & $9 / 10$ \\
\hline $40-59$ & $52.0 \pm 4.3$ & $-1.9 \pm 2.1$ & $22(20)$ & $9 / 13$ \\
\hline $60-79$ & $65.8 \pm 4.7$ & $-0.8 \pm 2.4$ & $27(21)$ & $11 / 16$ \\
\hline Note: SE: spherical equivalents; OD: oculus dexter; OS: oculus sinister & \\
\hline
\end{tabular}

Table 1

b. Choroidal thicknesses in different age groups predicted by proposed deep learning model

\begin{tabular}{|lllll|}
\hline Age group (years) & Mean choroidal thickness $(\mu \mathrm{m})$ & \multicolumn{2}{l}{$95 \% \mathrm{Cl}(\mu \mathrm{m})$} & \multirow{2}{*}{$p$ value } \\
\cline { 3 - 4 } & & Lower bound & Upper bound & \\
\hline $20-39(\mathrm{n}=19)$ & $253.7 \pm 41.9$ & 233.4 & 273.9 & $<0.01$ \\
\hline $20-59(\mathrm{n}=22)$ & $206.8 \pm 35.4$ & 191.0 & 222.5 & \\
\hline $60-79(\mathrm{n}=27)$ & $152.5 \pm 45.7$ & 134.5 & 170.6 & \\
\hline
\end{tabular}

Table 1

c. Choroidal thickness in different age groups calculated using physician sketches

\begin{tabular}{|c|c|c|c|c|}
\hline \multirow[t]{2}{*}{ Age group (years) } & \multirow[t]{2}{*}{ Mean choroidal thickness $(\mu \mathrm{m})$} & \multicolumn{2}{|l|}{$95 \% \mathrm{Cl}(\mu \mathrm{m})$} & \multirow[t]{2}{*}{$p$ value } \\
\hline & & Lower bound & Upper bound & \\
\hline $20-39(n=19)$ & $312.4 \pm 49.4$ & 288.6 & 336.2 & \multirow[t]{3}{*}{$<0.01$} \\
\hline $20-59(n=22)$ & $219.6 \pm 35.7$ & 203.8 & 235.5 & \\
\hline $60-79(n=27)$ & $179.2 \pm 48.8$ & 159.8 & 198.5 & \\
\hline
\end{tabular}

Note: $\mu \mathrm{m}$ : micrometer; Cl: confidence interval

Table $1 \mathrm{~b}$ lists the mean choroidal thicknesses predicted by our Mask R-CNN model. The 20-39-year group had the largest predicted mean choroidal thickness $(253.7 \pm 41.9 \mu \mathrm{m})$, followed by the $40-59$-year group $(206.8 \pm 35.4 \mu \mathrm{m})$. The $60-79$-year group had the lowest choroidal thickness $(152.5 \pm 45.7 \mu \mathrm{m})$. In 
each age group, normal distribution was identified by the Shapiro-Wilk test ( $p=0.14,0.73$, and 0.80 , in the 20-39-, 40-59-, and 60-79-year groups, respectively). Our ANOVA results revealed significant differences in mean choroidal thickness between the three groups $(p<0.01)$. Furthermore, a post hoc analysis revealed a statistical difference in choroidal thickness between any two groups (all $p<0.01$ ). Table 1c lists the mean choroidal thicknesses measured on the basis of physician sketches. The mean choroidal thicknesses were $312.4 \pm 49.4,219.6 \pm 35.7$, and $179.2 \pm 48.8 \mu \mathrm{m}$ in the $20-39-, 40-59-$, and 60-79-year groups, respectively, indicating statistically significant differences. Compared with the manually sketched contours, the average error obtained for the automatic predictions by our proposed Mask R-CNN model was 8.56 pixels.

The linear regression revealed a significant negative correlation between age and the automatically predicted mean choroidal thickness $\left(\mathrm{R}^{2}=0.53, p<0.01\right)$. Figure 2 presents a scatterplot of the mean choroidal thickness by age, which shows that choroidal thickness decreases as age increases. The regression formula is expressed as follows: mean choroidal thickness $(\mu \mathrm{m})=337.7-2.7 \times$ age. In addition, we observed similar trends for the choroidal thickness measured on the basis of the manual sketches; that is, the mean choroidal thickness increased in the older age group $(p<0.01)$, and a negative linear correlation was revealed between age and the derived mean choroidal thickness $\left(R^{2}=0.59, p<\right.$ $0.01)$.

We performed further analysis to establish the relationship between choroidal thickness and model prediction accuracy. Figure 3 presents a scatterplot and box plot of prediction error versus choroidal thickness, with the cutoff point being set at $280 \mu \mathrm{m}$ based on the dataset labelled by physicians. The average prediction error was $6.49 \pm 3.57$ pixels in subjects with choroidal thicknesses of less than 280 $\mu \mathrm{m}(\mathrm{n}=49)$ and $13.90 \pm 7.94$ pixels in those with choroidal thicknesses of greater than $280 \mu \mathrm{m}(\mathrm{n}=19, p$ $<0.01$ ). As indicated by the box plot in Figure $3 a$, the proposed model achieved lower and less variable prediction errors in subjects with thinner choroids. Moreover, we observed a positive linear correlation between prediction error and choroidal thicknesses of greater than $280 \mu \mathrm{m}\left(\mathrm{R}^{2}=0.21, p=0.048\right.$; Figure $3 b)$. No significant relationship was observed between prediction error and choroidal thicknesses of less than $280 \mu \mathrm{m}$ and $\left(R^{2}=0.01, p=0.54\right)$. Figure 4 demonstrates the choroidal contours delineated manually by physicians and those predicted by our model for two participants. One of the two participants had a thinner choroid, and the prediction error was 4.88 pixels; the other participant had a thicker choroid, and the prediction error was higher.

In our study, only subjects without high myopia or hyperopia were included. In the situation of SE refraction error between -6.0 and $+6.0 \mathrm{D}$, no significant correlation was identified between choroidal thickness and SE $\left(\mathrm{R}^{2}=0.04, p=0.11\right)$.

\section{Discussion}

The major findings of this study are outlined as follows: (1) The mean choroidal thickness reduced significantly for every 20-year increase in age. (2) A negative correlation was demonstrated between age 
and choroidal thickness. (3) Mask R-CNN model achieved satisfactory prediction accuracy in subjects with choroidal thicknesses of less than $280 \mu \mathrm{m}$.

We established that choroidal thickness decreased as age increased in subjects without significant ocular diseases or a history of intraocular operation. This finding is consistent with those of previous studies including Japanese, Korean, Chinese, and Iranian populations [8-10, 16, 17]. As to other factors, refractive error is considered to be negatively correlated with choroidal thickness. However, our study revealed no significant correlation between refractive error and choroidal thickness in our participants, for whom the refractive error was between -6 and $+6 \mathrm{D}$. This finding is in line with the results of Fujiwara et al. $\left(p=0.10, \mathrm{R}^{2}=0.1\right)[19]$. Furthermore, on the basis of a multiple regression analysis, lkuno et al. reported that axial length was not significantly associated with choroidal thickness $(p=0.22)$ [16].

For our Mask R-CNN model predictions, the mean choroidal thickness was $198.3 \pm 58.4 \mu \mathrm{m}$, which was lower than that obtained using physician sketches $(229.5 \pm 70.6 \mu \mathrm{m})$. The error observed for our model (8.56 pixels) is slightly higher than the average error (4.56 pixels) observed for a previously proposed model [15]. We noticed found that the prediction error was higher in the 20-39-year group, with thicker choroids, than in the other two groups. Figure 4 also depicts the difficulty in accurately delineating the choroidal outer boundary around the CSI when a thicker choroid is presented. We speculate that prediction accuracy could be affected in cases with thicker choroids. Possible reasons are that the quality and visibility of the CSI could be poorer in images of thicker choroids. In addition, the training and testing datasets comprised images of subjects aged from 21 to 79 years, for whom the choroidal thickness and morphology varied; such variations might engender challenges in accurately predicting the choroidal thickness. Further analysis demonstrated that the average prediction error observed for subjects with choroidal thicknesses of more than $280 \mu \mathrm{m}$ was significantly higher and more variable than that observed for those with choroidal thicknesses of less than $280 \mu \mathrm{m}$. Specifically, the prediction error was 6.49 pixels in subjects with choroidal thicknesses of less than $280 \mu \mathrm{m}$, and this value was not related to the change in choroidal thickness. This finding indicates that the proposed deep learning model is reliable and applicable under this condition. However, in subjects with choroidal thicknesses of greater than 280 $\mu \mathrm{m}$, the prediction error varied, and prediction error was positively correlated with choroidal thickness. This demonstrates that the proposed model is more suitable for images from subjects with choroidal thicknesses that do not exceed $280 \mu \mathrm{m}$. Previous studies have demonstrated that the successful measurement rate decreased as SFCT increased. A previous study reported that enhanced depth imaging (EDI)-OCT [20] appeared to improve the successful measurement rate, but obvious differences existed between thinner and thicker choroids [21]. Therefore, for subjects with extremely thick choroids and pachychoroid diseases, such as PCV or CSCR, their findings and data should be interpreted cautiously.

The choroid is stratified into three layers: the choriocapillaris with small vessels in the superficial layer; Sattler's layer with medium-sized vessels in the middle; and Haller's layer, the outer layer with large vessels. Choroidal morphology and thickness are influenced by not only physiological changes but also pathological factors [22]. Tissue water content and vascular endothelial growth factor (VEGF) are possible factors contributing to reduced choroidal thickness in healthy elderly people. A previous study 
that applied a water-drinking test revealed that choroidal thickness expanded when the amount of water in the body increased [23]. Considering that the amount and proportion of water in the body gradually decrease with age, this may explain the findings in our study. In addition, The RPE secretes VEGF toward the basal side of the choroid, and it plays an essential role in choroidal development [24]. The VEGF receptors are located in the choriocapillaris. Previous studies have reported that as age increases, the diameter of the choriocapillaris and thickness of the choroid shrink while the thickness of Burch's membrane increases $[25,26]$. The accumulation of lipid content with age is considered to cause the thickening of Bruch's membrane, which possibly occludes the movement of water-soluble agents between the RPE and the choroid. Reduced VEGF secretion into the choriocapillaris may lead to the shrinking of the choroid. In addition to normal aging physiology, the alternation of the choroid has been reported in chorioretinal disease; for example, Haller's layer, Sattler's layer, and choroidal volume or thickness have been reported to be significantly decreased in certain patients with diabetes mellitus or AMD [27, 28]. Reduced choroidal thickness was also noted in retinitis pigmentosa [29].

Previous studies have reported several approaches to evaluating choroidal thickness in different age groups. Such approaches mainly involve manual measurements of choroidal thickness, including SFCT, at a single site or at sites located at different distances (e.g., 1 or $3 \mathrm{~mm}$ ) from the fovea superiorly, inferiorly, temporally, and nasally. SFCT is generally the highest among the measured sites $[9,10,16,17]$. However, the mentioned approaches have several potential limitations; for example, they cannot be used to fully evaluate the appearance and shape of the choroid, and the overall thickness might be miscalculated in a discontinuous or unsmooth choroidal contour. To conduct a thorough examination of choroidal topographic features, Ouyang et al. proposed choroidal thickness mapping under the concept of spatial distribution using SD-OCT [30]. Hirata et al. introduced a swept-source OCT (SS-OCT) approach for choroidal volume mapping [31]. Furthermore, Chhablani et al. reported that EDI-OCT exhibited high repeatability and reproducibility in manual choroidal volume measurements [32]. Choroidal spatial distribution indices (CSDIs), derived from choroidal volume, were proposed for quantifying the choroidal topographic distribution [33].

Some of the aforementioned methods measure only specific sites of the choroid rather than the entire layer, and others require manual choroidal segmentation. This might be a time-consuming and operatordependent process, making it difficult to build a relatively large population database. Our proposed Mask R-CNN model is advantageous because it is based on deep learning. Our model estimates choroidal thickness by measuring numerous continuous points through automatic segmentation, thus providing an extensive and intact viewpoint for evaluation, in contrast to previously reported approaches. In addition, automatic segmentation and measurement with high accuracy can facilitate the establishment of larger and more extensive databases more rapidly and consistently.

The present study has certain limitations. First, the number of subjects in each group was not high. Further research with a larger sample size might provide more comprehensive analyses, such as analyses stratified by each decade of age. Second, especially in for thicker choroids, precisely delineating the 
choroidal boundary was challenging. Lower predictive accuracy in thicker choroids has been reported. EDI-OCT and SS-OCT may address this problem [34-36].

The application of the Mask R-CNN model revealed the relationship between choroidal thickness and age in this study. Through this model, future studies can compare choroidal morphologies in different axial length or in various ocular diseases, such as AMD, PCV, and CSCR. In combination with the deep learning model, further evaluation might be examined automatically, such as choroidal volume mapping, CSDI, or choroidal vascularity index assessment $[37,38]$. In this way, the choroid can be evaluated using detailed and robust information rapidly and reliably.

Overall, by utilizing the Mask R-CNN model for choroidal boundary depiction, our study revealed that the mean choroidal thickness decreased with age, indicating a negative correlation with age. The model achieved acceptable predictive accuracy, especially in thinner choroids. We anticipate applying this efficient model in further research with a larger sample size and more heterogeneous population.

\section{Declarations}

\section{Statements and Declarations:}

All authors have no relevant financial or non-financial interests to disclose. No funding was received for conducting this study.

Competing interests:All authors have no relevant financial or non-financial interests to disclose.

Funding: No funding was received for conducting this study.

\section{References}

1. Linsenmeier RA, Padnick-Silver L (2000) Metabolic Dependence of Photoreceptors on the Choroid in the Normal and Detached Retina.Invest Ophthalmol Vis Sci41:3117-3123

2. Parver LM, Auker C, Carpenter DO (1980) Choroidal blood flow as a heat dissipating mechanism in the macula. Am J Ophthalmol 89:641-646

3. Grossniklaus HE, Green WR (2004) Choroidal neovascularization. Am J Ophthalmol 137:496-503

4. Spaide RF, Hall L, Haas A et al (1996) Indocyanine green videoangiography of older patients with central serous chorioretinopathy. Retina Phila Pa 16:203-213

5. Flitcroft DI, He M, Jonas JB et al (2019) IMI - Defining and Classifying Myopia: A Proposed Set of Standards for Clinical and Epidemiologic Studies. Invest Ophthalmol Vis Sci 60:M20-M30

6. Polypoidal choroidal vasculopathy and treatments. https://doi.org/10.1097/ICU.0b013e3282fb7c33

7. Cheung CMG, Lee WK, Koizumi H et al (2019) Pachychoroid disease. Eye 33:14-33

8. Wakatsuki Y, Shinojima A, Kawamura A, Yuzawa M (2015) Correlation of Aging and Segmental Choroidal Thickness Measurement using Swept Source Optical Coherence Tomography in Healthy 
Eyes. PLoS ONE 10:e0144156

9. Entezari M, Karimi S, Ramezani A et al (2018) Choroidal Thickness in Healthy Subjects. J Ophthalmic Vis Res 13:39-43

10. Ding X, Li J, Zeng J et al (2011) Choroidal Thickness in Healthy Chinese Subjects. Invest Ophthalmol Vis Sci 52:9555-9560

11. Tian J, Marziliano P, Baskaran M et al (2013) Automatic segmentation of the choroid in enhanced depth imaging optical coherence tomography images. Biomed Opt Express 4:397-411

12. Masood S, Fang R, Li P et al (2019) Automatic Choroid Layer Segmentation from Optical Coherence Tomography Images Using Deep Learning. Sci Rep 9:3058

13. He F, Chun RKM, Qiu Z et al (2021) Choroid Segmentation of Retinal OCT Images Based on CNN Classifier and I2-Iq Fitter. Comput Math Methods Med 2021:8882801.

https://doi.org/10.1155/2021/8882801

14. He K, Gkioxari G, Dollar P, Girshick R (2017) Mask R-CNN. pp 2961-2969

15. Hsia WP, Tse SL, Chang CJ, Huang YL (2021) Automatic Segmentation of Choroid Layer Using Deep Learning on Spectral Domain Optical Coherence Tomography. Appl Sci 11:5488

16. Ikuno Y, Kawaguchi K, Nouchi T, Yasuno Y (2010) Choroidal Thickness in Healthy Japanese Subjects. Invest Ophthalmol Vis Sci 51:2173-2176

17. Kim M, Kim SS, Koh HJ, Lee SC (2014) Choroidal thickness, age, and refractive error in healthy Korean subjects. Optom Vis Sci Off Publ Am Acad Optom 91:491-496

18. Russell BC, Torralba A, Murphy KP, Freeman WT (2005) LabelMe:a database and web-based tool for image annotation

19. Fujiwara A, Shiragami C, Shirakata Y et al (2012) Enhanced depth imaging spectral-domain optical coherence tomography of subfoveal choroidal thickness in normal Japanese eyes. Jpn J Ophthalmol 56:230-235

20. Spaide RF, Koizumi H, Pozzoni MC, Pozonni MC (2008) Enhanced depth imaging spectral-domain optical coherence tomography. Am J Ophthalmol 146:496-500

21. Kong M, Choi DY, Han G et al (2018) Measurable Range of Subfoveal Choroidal Thickness With Conventional Spectral Domain Optical Coherence Tomography. Transl Vis Sci Technol 7:16

22. Zhao J, Wang YX, Zhang Q et al (2018) Macular Choroidal Small-Vessel Layer, Sattler's Layer and Haller's Layer Thicknesses: The Beijing Eye Study. Sci Rep 8:4411

23. Mansouri K, Medeiros FA, Marchase N et al (2013) Assessment of Choroidal Thickness and Volume during the Water Drinking Test by Swept-Source Optical Coherence Tomography. Ophthalmology 120:2508-2516

24. Marneros AG, Fan J, Yokoyama Y et al (2005) Vascular Endothelial Growth Factor Expression in the Retinal Pigment Epithelium Is Essential for Choriocapillaris Development and Visual Function. Am J Pathol 167:1451-1459 
25. Blaauwgeers HGT, Holtkamp GM, Rutten H et al (1999) Polarized Vascular Endothelial Growth Factor Secretion by Human Retinal Pigment Epithelium and Localization of Vascular Endothelial Growth Factor Receptors on the Inner Choriocapillaris: Evidence for a Trophic Paracrine Relation. Am J Pathol 155:421-428

26. Ramrattan RS, van der Schaft TL, Mooy CM et al (1994) Morphometric analysis of Bruch's membrane, the choriocapillaris, and the choroid in aging.Invest Ophthalmol Vis Sci35:2857-2864

27. Foo VHX, Gupta P, Nguyen QD et al (2020) Decrease in Choroidal Vascularity Index of Haller's layer in diabetic eyes precedes retinopathy. BMJ Open Diabetes Res Care 8:e001295

28. Esmaeelpour M, Ansari-Shahrezaei S, Glittenberg C et al (2014) Choroid, Haller's, and Sattler's Layer Thickness in Intermediate Age-Related Macular Degeneration With and Without Fellow Neovascular Eyes. Invest Ophthalmol Vis Sci 55:5074-5080

29. Dhoot DS, Huo S, Yuan A et al (2013) Evaluation of choroidal thickness in retinitis pigmentosa using enhanced depth imaging optical coherence tomography. $\mathrm{Br} \mathrm{J}$ Ophthalmol 97:66-69

30. Ouyang Y, Heussen FM, Mokwa N et al (2011) Spatial Distribution of Posterior Pole Choroidal Thickness by Spectral Domain Optical Coherence Tomography. Invest Ophthalmol Vis Sci 52:70197026

31. Hirata M, Tsujikawa A, Matsumoto A et al (2011) Macular Choroidal Thickness and Volume in Normal Subjects Measured by Swept-Source Optical Coherence Tomography. Invest Ophthalmol Vis Sci 52:4971-4978

32. Chhablani J, Barteselli G, Wang H et al (2012) Repeatability and Reproducibility of Manual Choroidal Volume Measurements Using Enhanced Depth Imaging Optical Coherence Tomography. Invest Ophthalmol Vis Sci 53:2274-2280

33. Hwang S, Kong M, Song Y-M, Ham D-I (2020) Choroidal spatial distribution indexes as novel parameters for topographic features of the choroid. Sci Rep 10:574

34. Copete S, Flores-Moreno I, Montero JA et al (2014) Direct comparison of spectral-domain and sweptsource OCT in the measurement of choroidal thickness in normal eyes. $\mathrm{Br} \mathrm{J}$ Ophthalmol 98:334-338

35. Lee M-W, Park H-J, Shin Y-I et al (2020) Comparison of choroidal thickness measurements using swept source and spectral domain optical coherence tomography in pachychoroid diseases. PLoS ONE 15:e0229134

36. Fujiwara T, Imamura Y, Margolis R et al (2009) Enhanced depth imaging optical coherence tomography of the choroid in highly myopic eyes. Am J Ophthalmol 148:445-450

37. Agrawal R, Gupta P, Tan K-A et al (2016) Choroidal vascularity index as a measure of vascular status of the choroid: Measurements in healthy eyes from a population-based study. Sci Rep 6:21090

38. Iovino C, Pellegrini M, Bernabei F et al (2020) Choroidal Vascularity Index: An In-Depth Analysis of This Novel Optical Coherence Tomography Parameter. J Clin Med 9:595

\section{Figures}




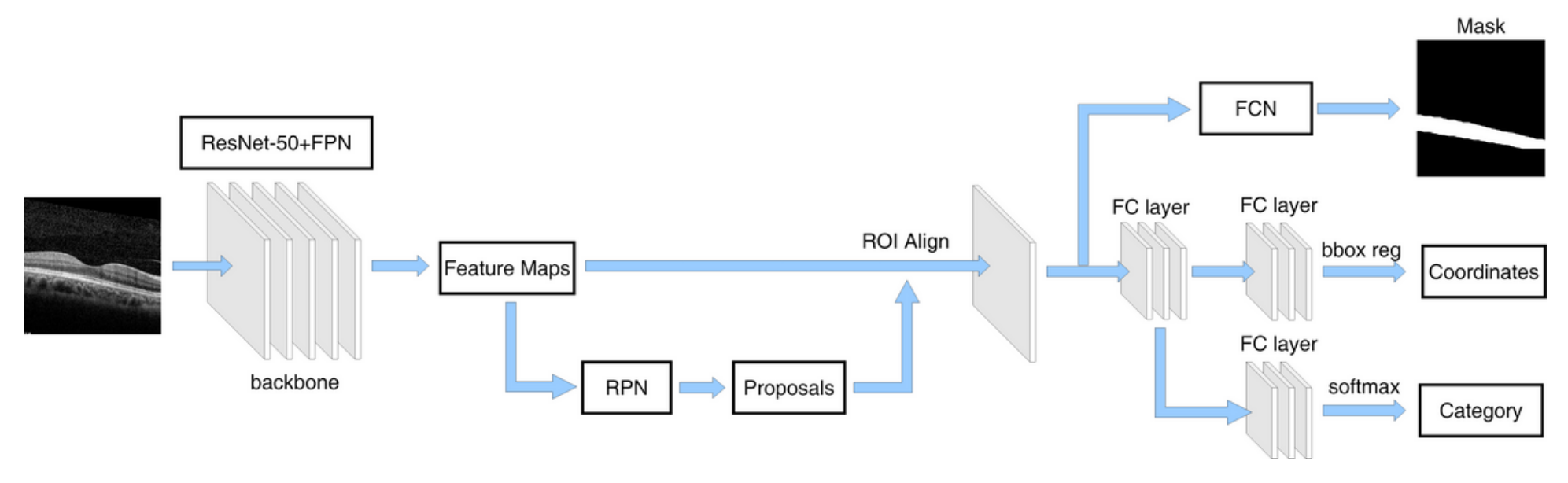

Figure 1

Framework of the proposed Mask R-CNN model. The OCT image is input into the backbone ResNet and FPN to generate feature maps. The RPN module is used to map the ROI onto the feature maps. A mask of the choroid is generated by the FCN. Note: ResNet: residual network; RPN: regional proposal network; ROI: region of interest; FCN: fully connected network; Mask R-CNN: mask region-based convolutional neural network. 


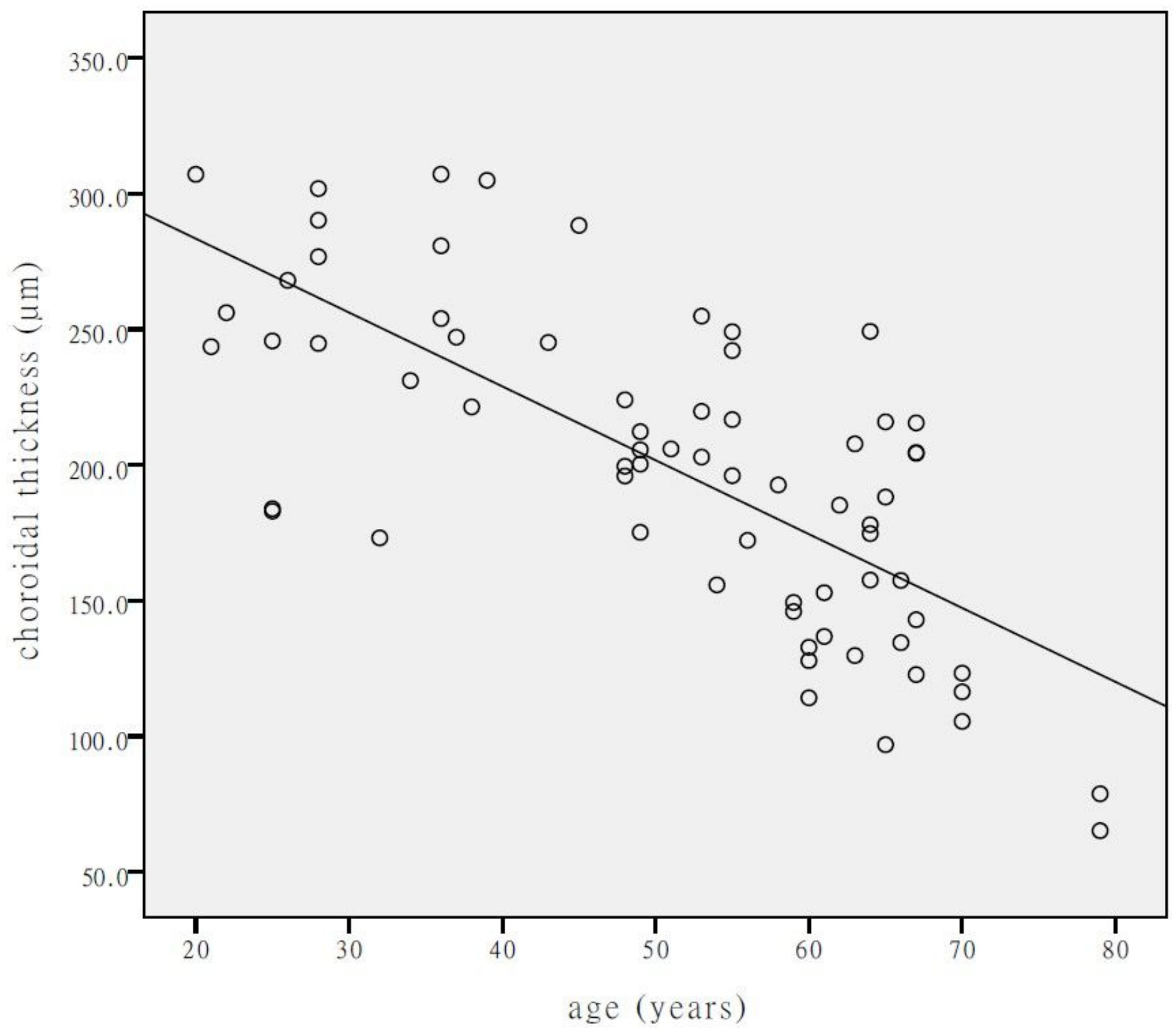

Figure 2

Correlations between age and mean choroidal thickness predicted by the proposed deep learning model $(p<0.01$, mean choroidal thickness $(\mu \mathrm{m})=-2.7 \times$ age $+337.7, \mathrm{R} 2=0.526)$. 


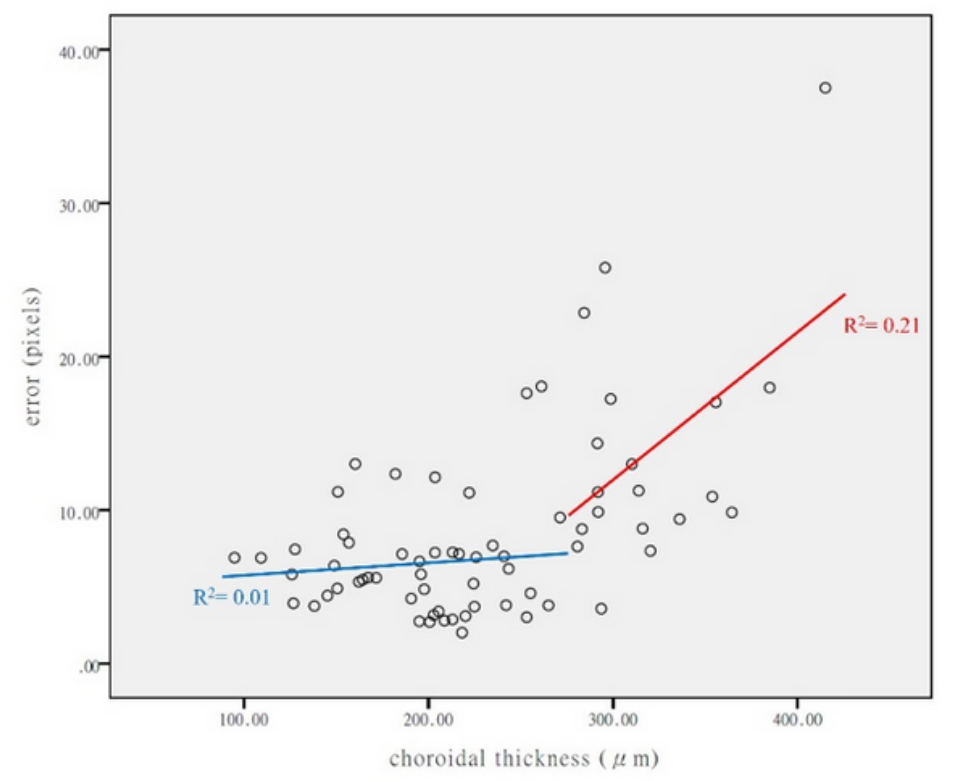

(a)

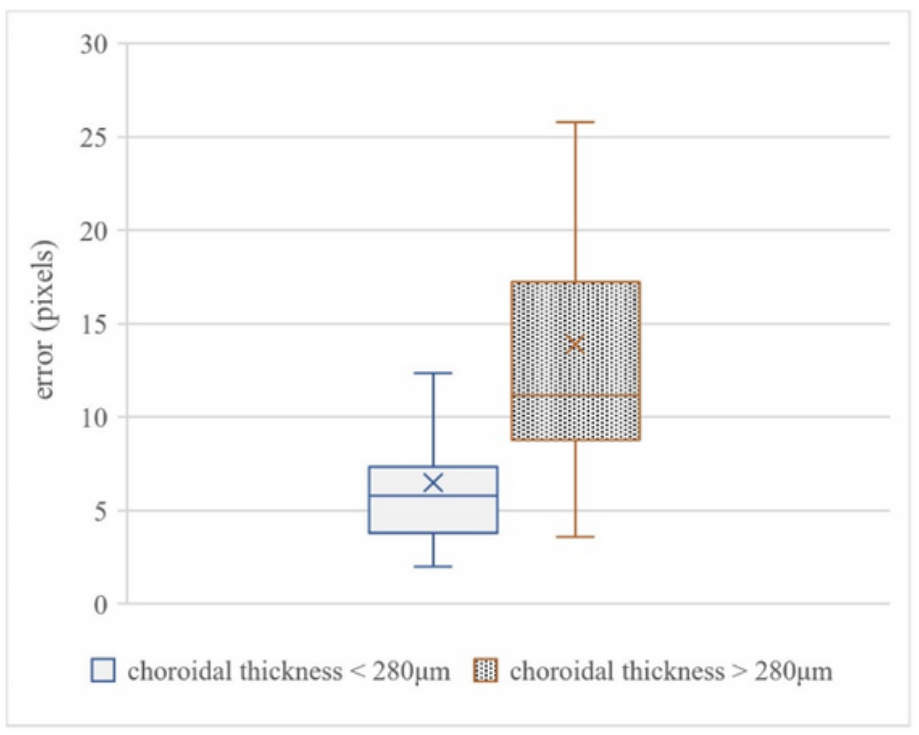

(b)

\section{Figure 3}

Relationship between prediction error and choroidal thickness for sketches manually labeled by a physician. The cutoff point for choroidal thickness was $280 \mu \mathrm{m}$. (a) Scatterplot of correlations between prediction error (pixels) and choroidal thickness $(R 2=0.01, p=0.54$ below the cutoff point; $R 2=0.21, p=$ 0.048 above the cutoff point). (b) Box plot of the prediction error (pixels); the left side depicts the prediction error (pixels) of choroidal thickness below the cutoff point $(280 \mu \mathrm{m} ; \mathrm{Q} 1=3.79, \mathrm{Q} 2=5.79, \mathrm{Q} 3=$ $7.33, \mathrm{n}=49)$; the right side depicts choroidal thickness above the cutoff point $(\mathrm{Q} 1=8.78, \mathrm{Q} 2=11.16, \mathrm{Q} 3$ $=17.23, \mathrm{n}=19)$. 


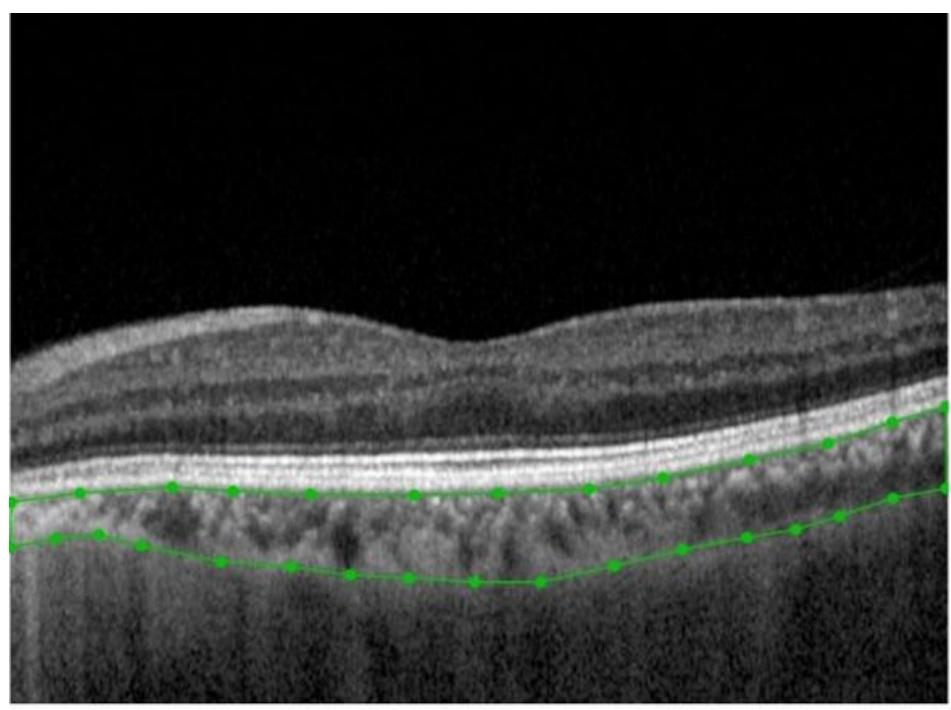

(a)

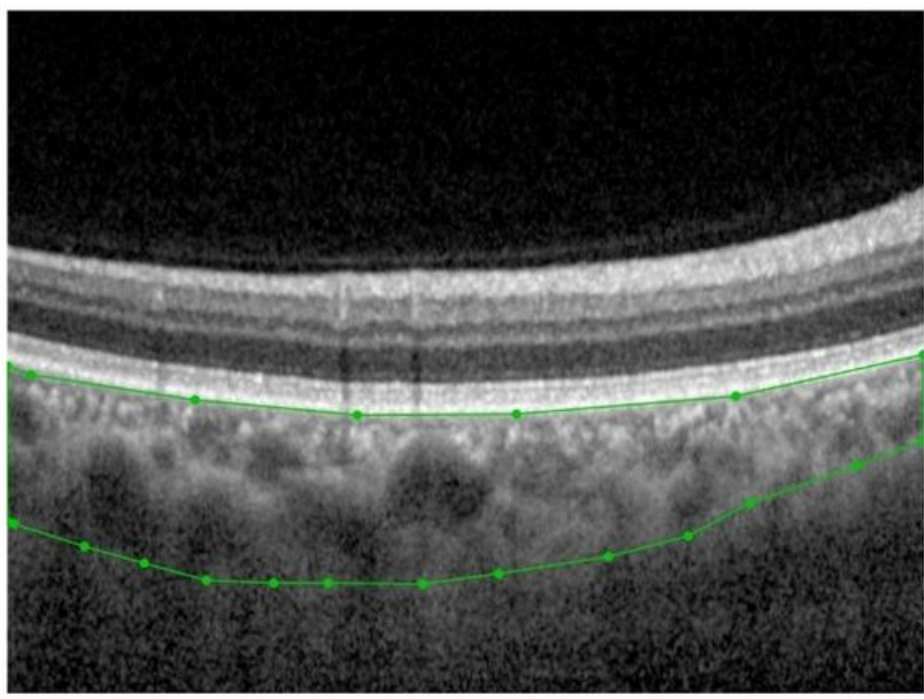

(c)

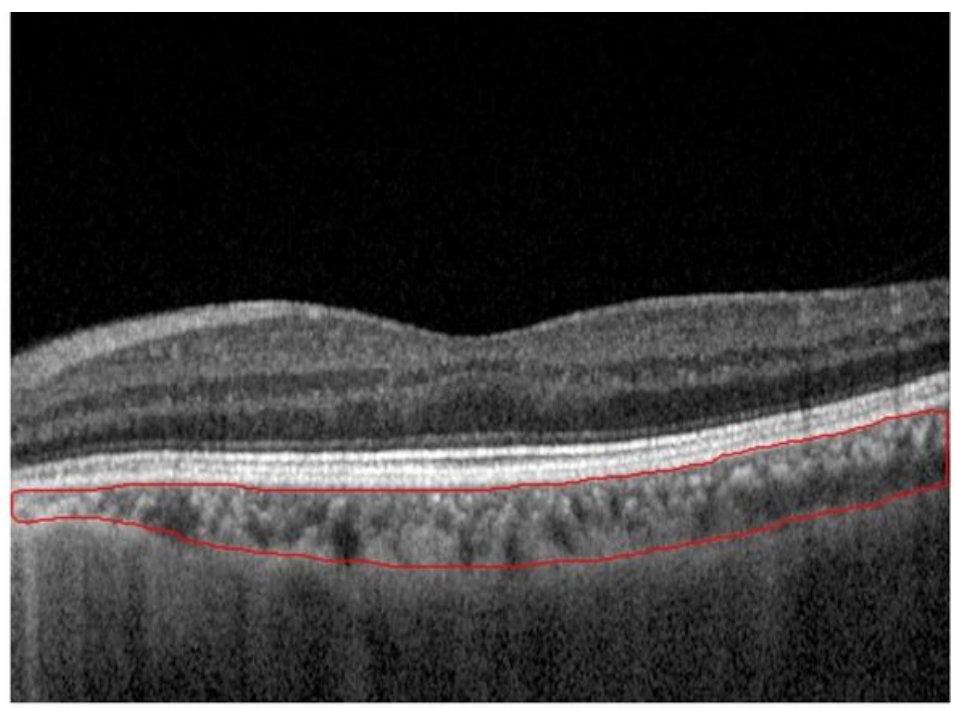

(b)

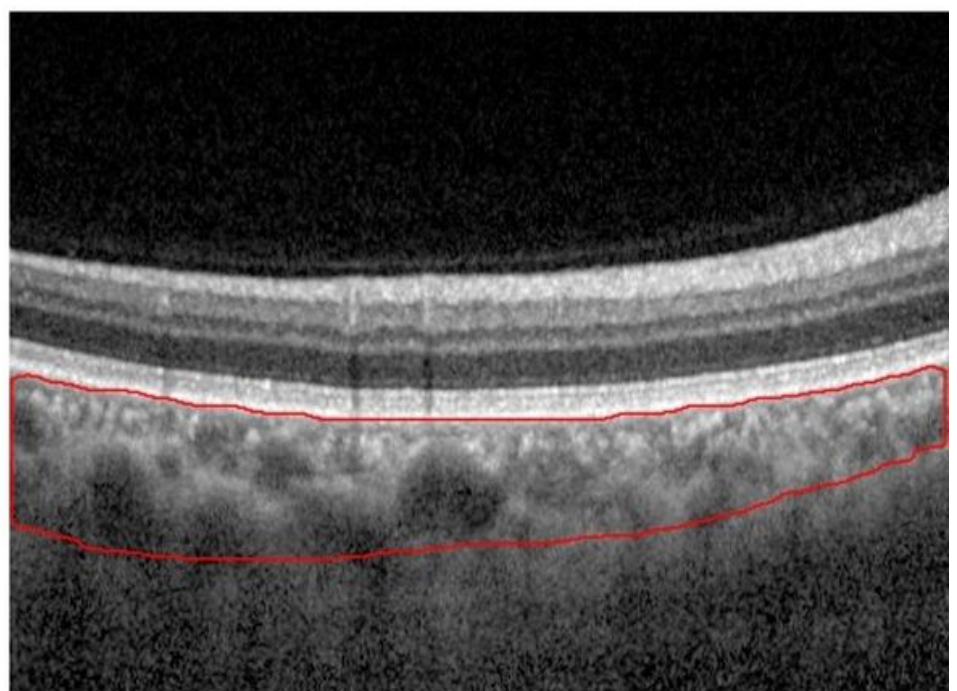

(d)

\section{Figure 4}

Delineation of the choroidal contour in two cases. Case 1: 61-year-old participant (error: 4.9 pixels). Case 2: 38-year-old participant (error: 17.2 pixels). (a) Green outline indicates the contour from physician sketch for case 1, with a mean choroidal thickness of $150.7 \mu \mathrm{m}$; (b) red outline represents the deep learning model prediction of case 1, with a mean choroidal thickness of $136.8 \mu \mathrm{m}$; (c) green outline indicates the contour from the physician's sketch of case 2, with a choroidal thickness of $298.7 \mu \mathrm{m}$; and (d) red outline represents the deep learning model prediction of case 2 , with a choroidal thickness of $221.3 \mu \mathrm{m}$. The relatively imprecise contour of the choroidal outer boundary is noted. 